\title{
Lügenpresse, schreibt die Presse? \\ Die Selbstthematisierung deutscher Tageszeitungen in Bezug auf den Begriff Lügenpresse
}

\author{
Nora Denner \& Christina Peter
}

\section{Abstract}

Seit mehreren Jahren sehen sich die klassischen Massenmedien zunehmend dem Vorwurf ausgesetzt, Bürgerinnen ${ }^{l}$ nicht mehr korrekt zu informierenLügenpresse und »Fake News« sind die Begriffe, die einem in diesem Zusammenhang in den Sinn kommen. Der vorliegende Beitrag beschäftigt sich mit der Frage, wie die Medien mit solchen Begriffen, speziell dem deutschen Begriff Lügenpresse, in ihrer Berichterstattung umgehen. Im Rahmen einer Inhaltsanalyse deutscher Tagezeitungen zeigte sich, dass der Begriff durchaus aktiv von Journalistinnen ins Spiel gebracht wird, ohne ihn jedoch konsequent zu reflektieren beziehungsweise dem dahinterstehenden Vorwurf zu begegnen.

Massenmedien gelten aufgrund ihrer Informations- und Orientierungsfunktion als eine zentrale Säule in modernen Demokratien (Ronneberger, 1974). Auch wenn ihnen je nach Demokratieverständnis unterschiedliche Rollen zugeschrieben werden (Jandura \& Friedrich, 2014), so herrscht doch Einigkeit bezüglich der zentralen Aufgabe, für Bürgerinnen eine gemeinsame, unabhängige Informationsbasis herzustellen, so dass sie informierte Entscheidungen treffen und entsprechend ihre Bürgerinnenpflichten wahrnehmen können. Um diese Aufgabe ausüben zu können, ist ein Mindestmaß an Vertrauen in die Medien und ihre Berichterstattung nötig. Gerade in den letzten Jahren wurden allerdings Zweifel laut, ob dies für die Mehrheit der Bevölkerung noch gegeben ist (Reinemann \& Fawzi, 2016). Auslöser für die - zumindest wahrgenommene - Vertrauenskrise waren vor allem rechtspopulistische Bewegungen, die unter dem Schlagwort Lügenpresse ihren Frust gegenüber den klassischen Massenmedien zum Ausdruck brachten

1 Aus Gründen der Lesbarkeit wird auf geschlechtsspezifische Differenzierung verzichtet und ausschließlich die weibliche Form verwendet. Die männliche Form ist dabei miteingeschlossen. 
(Neverla, 2017; Reinemann, Fawzi \& Obermaier, 2017). Das englische Äquivalent »Fake News«, das besonders der amtierende US Präsident Trump en vouge machte (Nelson \& Taneja, 2018), zielt ähnlich auf die Delegitimierung der sogenannten Mainstream-Medien als Lieferant valider Informationen.

Beide Begriffe - Lügenpresse und »Fake News« - scheinen allgegenwärtig, und dies liegt nicht allein daran, dass man Donald Trump auf Twitter folgt oder Pegida-Demonstrationen in der Innenstadt beobachtet. Die Begriffe begegnen einem vor allem deshalb, weil die Medien die Begriffe reproduzieren - sei es durch eine bloße Darstellung, zum Beispiel auf Bildern von Demonstrationen, oder durch eine kritische Auseinandersetzung mit den dahinterstehenden Vorwürfen. Um diesen Selbstthematisierungsaspekt der Massenmedien soll es in diesem Beitrag vornehmlich gehen. Wir wollen der Frage nachgehen, wie die Medien mit dem Begriff Lügenpresse umgehen, ob und wie sie den darin enthaltenen Vorwürfen begegnen und welche Konsequenzen der Umgang damit letztendlich für die Wahrnehmung und das Vertrauen der Leserinnen in die Medien haben könnte.

Bevor wir uns der Selbstthematisierung der Medien in Zusammenhang mit dem Begriff Lügenpresse widmen, sind aus unserer Sicht zunächst zwei Einordnungen wichtig. Zum einen wollen wir uns zunächst dem Begriff und seinen Synonymen widmen. Dabei sollen deren Bedeutung und die hinter der Verwendung stehende Problematik, nämlich der Ausdruck von Misstrauen in die Massenmedien, diskutiert werden. Zum anderen scheint es uns aufbauend darauf wichtig, zu eruieren, ob es eine oft im Zusammenhang mit den Begriffen postulierte Vertrauenskrise der Massenmedien so überhaupt gibt.

Lügenpresse, »Fake News« \& Co. - Wer traut noch wem?

Obwohl Lügenpresse als Begriff vor allem in den letzten Jahren im Kontext von rechtspopulistischen Bewegungen einen Aufschwung erlebte, ist seine Verwendung sowie der dahinterstehende Vorwurf kein neues Phänomen. Bereits im ersten Weltkrieg diente der Begriff dazu, ausländische Medien zu diffamieren (Heine, 2015). Auch wurde er keineswegs nur vom rechten Rand verwendet, sondern allgemein antidemokratisch aufgeladen und von verschiedenen politischen Lagern als Diffamierung anders gesinnter Medien gebraucht (Heine, 2015). Diesen Zweck erfüllt der Begriff auch noch heute, auch wenn er sich pauschaler an die klassischen, sogenannten Mainstream-Medien richtet. Ziel ist es, diesen Medien gezielt die 
Deutungshoheit über den politischen Diskurs und damit die Informationsbeziehungsweise Vermittlungsfunktion zwischen Öffentlichkeit und politischer Elite abzusprechen. In der Begrifflichkeit schwingt dabei der Vorwurf an den Journalismus mit, die Bevölkerung in vollem Bewusstsein falsch zu informieren - denn lügen bedeutet, dass Unwahrheiten absichtlich verbreitet werden (Hagen, 2015).

Während der deutsche Begriff Lügenpresse dabei eindeutig als Vorwurf gegen die klassischen Massenmedien zu verstehen ist, ist das englische Äquivalent $»$ Fake News« mehrdeutig. Egelhofer und Lecheler (2019) unterscheiden in ihrer Typologisierung zwischen der Verwendung des Begriffs als Vorwurf gegen die Massenmedien (fake news as label) auf der einen Seite (und damit äquivalent zum Begriff Lügenpresse) und einem speziellen Desinformationstyp auf der anderen Seite (fake news as genre). Bei letzterem handelt es sich um Falschinformationen, die aus politischen oder ökonomischen Motiven absichtlich verbreitet werden und dabei echte journalistische Beiträge imitieren (Zimmermann \& Kohring, 2018). Die Urheberinnen der Falschmeldungen machen sich hier also bewusst den Vertrauensvorschub zunutze, der dem klassischen Journalismus entgegengebracht wird. Daran wird das ganze Dilemma der Vermischung der Begrifflichkeiten klar: Während die Verbreiterinnen von echten »Fake News« versuchen, vom Vertrauen in den Journalismus zu profitieren und ihre Angebote entsprechend gestalten, versuchen Kritikerinnen mit dem Vorwurf »Fake News« wiederum, Inhalte klassischer Massenmedien auf die gleiche Stufe wie gezielte Falschinformationen zu stellen.

Zusätzlich wird es in modernen Medienumgebungen für die Rezipientinnen immer schwieriger, Fakten und Falschinformationen zu unterscheiden (Tandoc, Lim \& Ling, 2018). Durch das Internet im Allgemeinen und soziale Medien im Speziellen kann sich prinzipiell jede Information mit variierendem Wahrheitsgehalt einfach, schnell und kostengünstig verbreiten (Allcott \& Gentzkow, 2017). Gezielte Falschnachrichten sind meist in Form von Nachrichten-Websites aufbereitet und werden hauptsächlich über soziale Netzwerke verbreitet (Müller \& Denner, 2019), erscheinen damit also im schlimmsten Fall neben klassischen Nachrichtenartikeln in der Facebook-Timeline. Vor allem Falschmeldungen, die von befreundeten Personen in sozialen Medien weitergeleitet werden, werden dabei seltener auf ihren Wahrheitsgehalt hin überprüft (Torres, Gerhart \& Negahban, 2018). Hinzu kommen Akteurinnen, die unter anderem über soziale Netzwerke mit Lügenpresse- oder »Fake News«-Vorwürfen gezielt versuchen, das Vertrauen in die Medien zu schwächen. 
Führt das alles zusammengenommen dazu, dass das Vertrauen in die Medien tatsächlich erodiert? Journalistinnen selbst scheinen es zumindest so wahrzunehmen - Begriffe wie »Vertrauenskrise« oder Aussagen wie »das Vertrauen in die klassischen Massenmedien sinkt«, prägten in den letzten Jahren die Schlagzeilen und den politischen Diskurs (Reinemann \& Fawzi, 2016). Tatsächlich lassen sich diese Vermutungen, zumindest für Deutschland, so nicht bestätigen. Bereits 2016 zeigten Reinemann und Fawzi bei der Auswertung verschiedener empirischer Studien, dass das Medienvertrauen seit Jahrzehnten stabil ist (Reinemann \& Fawzi, 2016).

Die Mainzer Langzeitstudie zu Medienvertrauen kommt dabei zu ähnlichen Befunden (Jackob et al., 2019): Auch hier zeigt sich ein einigermaßen stabiles Bild beim Vertrauen in öffentlich-rechtlichen Rundfunk und Tageszeitungen, während das Vertrauen in das Internet als Quelle eher zurückgeht. Betrachtet man das Medienvertrauen global, fällt dieses 2018 so hoch aus wie noch nie: 44 Prozent der Befragten geben an, den Medien bei wichtigen Dingen eher oder voll und ganz zu vertrauen (zum Vergleich: 2008 waren es 29 Prozent). Tatsächlich ist es aber so, dass auch der Anteil derer, die den Medien eher nicht oder überhaupt nicht vertrauen, über die Zeit deutlich ansteigt, und zwar von neun Prozent im Jahr 2008 auf 22 Prozent in 2018. Was man also relativ deutlich beobachten kann, sind Polarisierungstendenzen: Der Anteil derer, die unentschieden sind und sich damit vertrauenstechnisch eher in der Mitte positionieren, wird kleiner. Entsprechend konstatierten die Autorinnen der Studie 2017 zwar, dass aufgrund der Minderheit an Personen mit starken Vorbehalten »die Lügenpresse-Hysterie der vergangenen Jahre übertrieben [war] « (Ziegele et al., 2018, 150), räumen aber auch ein: »(...) dennoch haben die »Lügenpresse«-Vorwürfe und sicherlich auch einige journalistische Fehlgriffe Spuren hinterlassen: Pauschale Kritik und Polemik haben sich in den vergangenen Jahren verfestigt« (Jackob et al., 2019, 212).

In diesem Zusammenhang stellt sich die Frage, wie die Massenmedien selbst mit dem Vorwurf Lügenpresse umgegangen sind und inwiefern sie möglicherweise zu der vermeintlichen Hysterie selbst beigetragen haben. Wird der Begriff lediglich reproduziert oder auch tiefer reflektiert, werden die Vorwürfe entkräftet oder möglicherweise sogar - bezugnehmend auf die oben angesprochenen journalistischen Fehlgriffe - für andere Medien geltend gemacht? 


\section{Lügenpresse im Spiegel - Selbstthematisierung der Medien}

Der Lügenpresse-Vorwurf zielt in den letzten Jahren, wie oben dargelegt, üblicherweise pauschal auf die klassischen beziehungsweise als sogenannter Mainstream bezeichneten Massenmedien. Wenn diese also den Begriff Lügenpresse verwenden, thematisieren sie sich damit zunächst einmal selbst. Selbstthematisierung der Medien wird als mediale Kommunikation über Medien verstanden und findet sich als Forschungsgegenstand vor allem im Bereich der Medienkritik (Krüger \& Müller-Sachse, 1998; Malik, 2004) beziehungsweise im Themenfeld der Kriegs- oder Krisenberichterstattung (Drentwett, 2009; Esser, 2008). Einige Autorinnen unterscheiden im Rahmen der Selbstthematisierung verschiedene Reflexionsgrade (Beuthner \& Weichert, 2005; Malik, 2004; Reinemann \& Huismann, 2007). Ein eher niedriger Reflexionsgrad (auch als Selbstreferenz bezeichnet) meint dabei eher unkritische Bezüge zur eigenen Profession beziehungsweise journalistischem Handeln. Ein hoher Reflexionsgrad bedeutet, dass die Medien sich kritisch mit ihrer eigenen Rolle in der Gesellschaft auseinandersetzen. Dabei geht es auch um Ansprüche, die an die Medien gestellt werden, sowie eine intensive Auseinandersetzung mit und Bewertung von Inhalten und Aufbereitungsformen. Dies wird als Selbstreflexion bezeichnet.

Innerhalb der Medien findet solche Kritik an der eigenen Profession vor allem im Bereich des Feuilleton oder anderen medienbezogenen Rubriken statt (zum Beispiel im Rahmen von Journalismus- oder Literaturkritik), aber auch - und für die Frage nach dem Umgang mit dem Lügenpressevorwurf zentral - im Rahmen der politischen Kommunikation. Hier hat sich für die Selbstthematisierung der Begriff der Metaberichterstattung etabliert (D'Angelo \& Esser 2014; Denner \& Peter, 2017; Drentwett 2009; Esser 2008). Anders als bei der Medienkritik ist hier in der Regel der Kommunikationsanlass ein anderer, nämlich eben ein politisches Thema, in dessen Kontext dann die Rolle der Medien reflektiert wird (zum Beispiel Kritik an der Berichterstattung anderer Medien im Rahmen der Flüchtlingskrise).

Der Lügenpresse-Vorwurf kann auf beide Arten behandelt werden: Zum einen ist es natürlich wahrscheinlich, dass der Begriff im Kontext der Berichterstattung über Pegida und Co. auftaucht, zum anderen kann der Vorwurf an sich auch zum Anlass genommen werden, die (eigene) journalistische Arbeitspraxis beziehungsweise journalistische Normen im Allgemeinen kritisch zu hinterfragen. Die darauf aufbauende Vermutung wäre, dass der Begriff im Rahmen der Politikberichterstattung im Kontext von anderen 
Kommunikationsanlässen eher nur genannt und nicht so tief reflektiert wird wie etwa im Rahmen der Medienkritik.

Ausgehend von dieser Fragestellung haben wir eine quantitative Inhaltsanalyse der fünf größten deutschen Tagezeitungen ${ }^{2}$ durchgeführt (Denner \& Peter, 2017). Als Untersuchungszeitraum wählten wir Oktober 2014 bis Oktober 2015, um den Umgang mit dem Begriff Lügenpresse vor und nach seiner Wahl zum Unwort des Jahres 2014 (veröffentlicht am 15. Januar 2015) untersuchen zu können. Codiert wurden alle Beiträge in diesem Zeitraum, in denen der Begriff Lügenpresse mindestens einmal vorkam, was bei 304 Beiträgen der Fall war. Davon entfielen 33 Prozent auf die taz, 29 Prozent auf die $F A Z$, gefolgt von der Welt mit 19 Prozent sowie der $S Z$ mit 16 Prozent. Die Bild wies mit Abstand am wenigsten Beiträge auf(3 Prozent).

Neben der Platzierung und Verwendungshäufigkeit pro Artikel haben wir für jede Nennung des Begriffs auf Aussageebene codiert, wer die eigentliche Urheberin des Begriffs war. Wenn also zum Beispiel eine Zeitung schrieb, dass Pegida-Demonstrantinnen Plakate mit dem Aufdruck Lügenpresse trugen, dann wurde Pegida als Urheberin codiert.

Außerdem erhoben wir, auf welche mediale Ebene sich die Verwendung des Begriffs Lügenpresse bezog, also ob er verwendet wurde, um das Mediensystem, die Medien oder den Journalismus als Ganzes zu beschreiben (Makrolevel), oder ob er sich auf Medienorganisationen oder Medieneinheiten (Mesolevel) oder auf (einzelne) Medienakteurinnen (Mikrolevel) bezog.

Darüber hinaus ging es uns um die Beschäftigung mit dem Begriff im Kontext seiner Nennung, also den Reflexionsgrad: Wir wollten wissen, ob der Begriff lediglich genannt beziehungsweise reproduziert wird, oder ob eine tiefergehende Auseinandersetzung damit erfolgt. Aufbauend auf bisherigen Arbeiten zur Selbstthematisierung unterschieden wir, ob ein (unkritischer) Selbstbezug hergestellt wurde (Selbstreferenz) oder sogar eine tiefergehende Reflexion (Selbstreflexion) erfolgte. Eine Selbstreferenz wurde codiert, wenn der Begriff genannt und hergeleitet wurde. Damit ist gemeint, dass sich die Aussage entweder auf vorangegangene Berichterstattung oder Ereignisse im medialen Zusammenhang bezog, dabei allerdings nicht in einen übergeordneten Kontext eingeordnet oder die Rolle der Medien erörtert beziehungsweise reflektiert wurde. Als Selbstreflexion wiederum wurde codiert, wenn der Begriff beziehungsweise der dahinterstehende Vorwurf tiefergehend diskutiert wurde und eine kritische Auseinandersetzung tung (FAZ), die Welt, die tageszeitung (taz) sowie die Bild und die dazugehörigen Sonntagsausgaben. 
erfolgte - etwa in Bezug auf den konkreten Berichterstattungsanlass (zum Beispiel Verfehlung von Journalistinnen) oder auch ganz generell Aspekte wie die Herkunft des Begriffs und seine Instrumentalisierung oder die Rolle der Medien beziehungsweise des Journalismus in der Gesellschaft. Darüber hinaus haben wir noch etwas differenzierter die Einordnung beziehungsweise Bewertung des Begriffs aus Sicht der Urheberin codiert.

Der Reliabilitätskoeffizient nach Holsti (1969) beträgt bei den formalen Kategorien 0.99 (Spannweite 0.90 bis 1.00 ) sowie bei den inhaltlichen Kategorien 0.86 (Spannweite 0.75 bis 1.00). Die Reliabilität in Bezug auf die Identifikation der zu codierenden Akteursaussagen beträgt 1.00.

Insgesamt tauchte der Begriff etwas häufiger in Politik-Ressorts (39 Prozent) als in Medien-Ressorts (27 Prozent) auf, der Rest der Nennungen entfällt auf andere Ressorts (33 Prozent). Allgemein betrachtet zeigte sich dabei, dass der Begriff in 54 Prozent der Fälle lediglich genannt wird, in 30 Prozent zumindest weitergehend thematisiert und in nur rund 16 Prozent überhaupt tiefergehend reflektiert wird. Es bestätigte sich außerdem die Vermutung, dass der Begriff im Rahmen medienbezogener Ressorts öfter reflektiert wurde, also eine kritische Auseinandersetzung über einen konkreten Kommunikationsanlass hinaus erfolgte (bei rund 38 Prozent aller Verwendungen). Taucht der Begriff im Politik-Ressort auf, wurde er hingegen in etwas mehr als der Hälfte aller Fälle lediglich genannt, ohne dass auf den dahinterstehenden Vorwurf näher eingegangen wurde.

Betrachtet man den Reflexionsgrad nach Medium, zeigen sich zwischen den Qualitätszeitungen keine nennenswerten Unterschiede; lediglich die Bild verwendet den Begriff deutlich häufiger selbstreferenziell (in rund zwei Drittel aller Fälle), allerdings kommt der Begriff hier im Untersuchungszeitraum mit insgesamt zwölf Nennungen auch mit Abstand am seltensten vor. Am meisten reflektiert wird der Begriff in der FAZ (23 Prozent), gefolgt von der taz (16 Prozent), der Welt (12 Prozent), der SZ (11 Prozent) und der Bild (8 Prozent, das heißt in einem Verwendungsfall).

Seinen Höhepunkt in der Verwendung hatte der Begriff im Januar 2015, also kurz vor beziehungsweise nach der Wahl von Lügenpresse als Unwort des Jahres 2014. Allerdings stieg auch hier vor allem die Zahl der bloßen Nennungen, eine tiefere Auseinandersetzung mit dem Begriff fand also auch in diesem Kontext nur in geringem Maße statt (Denner \& Peter, 2017). 


\section{Weiterführende Befunde}

In diesem Kapitel werden einige weiterführende Befunde der Studie vorgestellt, die über die schon veröffentlichten Ergebnisse (Denner \& Peter, 2017) hinausgehen.

Betrachtet man zunächst die Platzierung und die Verwendungshäufigkeit des Begriffs Lügenpresse, zeigt sich Folgendes: Der Begriff wurde in zwölf Prozent der codierten Artikel das erste Mal in der Überschrift, Unterüberschrift oder im Teaser genannt, während er in 88 Prozent der Beiträge das erste Mal erst im Text vorkam. Die Anzahl der Nennungen pro Artikel variierte stark (Min. $\left.=1 ; \operatorname{Max} .=25^{3} ; M=1.48 ; S D=1.68\right)$, zwischen den verschiedenen Medien gab es allerdings keinen nennenswerten Unterschied. Das Gleiche gilt für verschiedene Stilformen und Ressorts.

Interessant war für uns auch, wer eigentlich als Urheberin des Begriffs fungierte. Tauchte dieser also hauptsächlich in der Berichterstattung auf, weil über die Verwendung durch Dritte berichtet wurde oder bringen die Medien den Begriff selbst ins Spiel? Die Auswertung zeigt, dass in mehr als der Hälfte der Fälle (55 Prozent) tatsächlich die Autorin des Zeitungsbeitrags den Ausdruck selbst aufbrachte und dieser nicht etwa deswegen auftauchte, weil externe Urheberinnen zitiert wurden. Die andere Hälfte verteilte sich auf eine Vielzahl von Akteurinnen, wobei die am häufigsten vorkommenden Pegida (13 Prozent), Politikerinnen (8 Prozent) und Journalistinnen anderer Printmedien waren (4 Prozent). Auch hier zeigten sich kaum Unterschiede zwischen den Zeitungen.

Betrachtet man in diesem Zusammenhang den Reflexionsgrad, zeigt sich zunächst ein signifikanter Unterschied zwischen Autorin des Beitrags und externer Urheberin des Begriffs $\left(X^{2}(2)=14.17, p<.01, \phi=.21 ; N=338\right)$ : Ist die Urheberin des Begriffs Lügenpresse auch Autorin des Beitrags, erfolgte häufiger eine tiefergehende Reflexion (in 20 Prozent aller Fälle), bei externen Urheberinnen war das etwas seltener der Fall (12 Prozent). Das bedeutet umgekehrt allerdings auch, dass in rund drei Viertel aller Fälle, in denen die Autorin des Beitrags den Begriff selbst ins Spiel brachte, sie ihn nur oberflächlich oder gar nicht reflektierte.

Die Analyse zeigt weiterhin, dass meistens das Mediensystem als Ganzes durch den Begriff Lügenpresse thematisiert wurde (80 Prozent aller Verwendungsfälle), was wahrscheinlich mit dem generell eher geringen Reflektionsniveau beziehungsweise der bloßen Reproduktion des Begriffs dem historischen Kontext beziehungsweise der Verwendung des Begriffs auseinandersetzt (Heine, 2015). 
zusammenhängt. Medienorganisationen oder Medieneinheiten wie zum Beispiel einzelne Sender, Zeitungen oder Verlagshäuser wurden mit neun Prozent und einzelne Journalistinnen mit elf Prozent eher weniger mit dem Begriff in Verbindung gebracht.

Betrachtet man in Verbindung damit die Reflexionstiefe, zeigt sich ein Unterschied zwischen den verschiedenen Bezugsebenen $\left(X^{2}(4)=19.26, p\right.$ $<.01, \phi=.24 ; N=338)$ : Wenn sich der Begriff Lügenpresse auf das Mediensystem beziehungsweise die Medien oder den Journalismus als Ganzes bezieht, wird der Begriff in mehr als der Hälfte der Fälle nur genannt (siehe Tabelle 1). ${ }^{4}$ Bezieht sich der Begriff auf einzelne Medienakteurinnen oder auf Medienorganisationen, wird der Begriff immerhin in drei Viertel der Fälle selbstreferenziell oder sogar selbstreflexiv betrachtet. Interessant ist, dass der Begriff am häufigsten in Bezug auf Medienorganisationen tiefer reflektiert wird (28 Prozent der Aussagen). Ein Beispiel dafür ist ein Gastbeitrag in der $F A Z$, in dem der Chefredakteur der $A R D$ auf die Vorwürfe, die $A R D$ würde einseitig berichten, eingeht (Gniffke, 2015).

Tabelle 1: Vergleich der Bezugsebene in Bezug auf den Grad der Selbstthematisierung (in Prozent)

\begin{tabular}{lcccc}
\hline $\begin{array}{l}\text { Grad der Selbst- } \\
\text { thematisierung }\end{array}$ & $\begin{array}{c}\text { Medien- } \\
\text { system }\end{array}$ & $\begin{array}{c}\text { Medienorga- } \\
\text { nisationen }\end{array}$ & $\begin{array}{c}\text { Medien- } \\
\text { akteurinnen }\end{array}$ & Gesamt \\
\hline Bloße Nennung & 59 & 24 & 37 & 54 \\
Selbstreferenz & 26 & 48 & 47 & 30 \\
Selbstreflexion & 15 & 28 & 16 & 16 \\
Gesamt & 100 & 100 & 100 & 100 \\
\hline
\end{tabular}

Anmerkungen: $N=338 ; X^{2}=19.26, p<.01, \phi=.24$

Zuletzt wurde analysiert, ob und wie der Begriff im Rahmen seiner Verwendung kontextualisiert beziehungsweise bewertet wurde. Dabei gilt es zu beachten, dass die Bewertung immer in Bezug zur Urheberin des Begriffs $\mathrm{zu}$ sehen ist.

Insgesamt betrachtet zeigt sich, dass der Begriff Lügenpresse in den Beiträgen mit 34 Prozent am häufigsten als Vorwurf an die Medien verwendet (und/oder als Schimpfwort bezeichnet) wurde (siehe Tabelle 2). In etwa 20 Prozent der Fälle wurde der Begriff überhaupt nicht kontextualisiert, in 24

4 Dies hängt allerdings auch damit zusammen, dass bei einer bloßen Nennung des Begriffs und keiner weiteren Information, auf wen sich der Begriff bezieht, das Mediensystem als Bezugsebene codiert wurde. 
Prozent wurde Lügenpresse als Synonym für die Medien beziehungsweise die Presse verwendet. Als unangebracht wurde der Begriff in acht Prozent der Aussagen bewertet, weil er aus dem Vokabular der Nationalsozialisten stammt. In vier Prozent wurde der Begriff als angebrachte Beschreibung der Medien aufgefasst. Sonstige Bewertungen kamen in zehn Prozent der Fälle vor.

Betrachtet man die Bewertung von Lügenpresse in Bezug auf die mediale Ebene, also ob sich der Begriff auf das Mediensystem, Medienorganisationen oder einzelne Akteurinnen bezieht, zeigten sich keine Unterschiede. Allerdings unterschied sich die Bewertung je nach Urheberin der Aussage (siehe Tabelle 2). War die Autorin des Beitrags die Urheberin, wird Lügenpresse häufiger als Synonym für die Medien verwendet als dies bei einer externen Urheberin der Fall war. Ebenso wurde der Begriff häufiger als unangebracht bezeichnet, weil er aus dem Vokabular der Nationalsozialisten entstammt. Externe Urheberinnen dagegen verwendeten den Begriff im Vergleich häufiger als Vorwurf beziehungsweise Schimpfwort.

Tabelle 2: Vergleich der Urheberinnen der Aussage (in Verbindung mit dem Begriff Lügenpresse) in Bezug auf die Bewertung des Begriffs (in Prozent)

\begin{tabular}{lccc}
\hline Art der Bewertung & Autorin & $\begin{array}{c}\text { Externe } \\
\text { Urheberin }\end{array}$ & Gesamt \\
\hline Keine Bewertung & 20 & 20 & 20 \\
\hline $\begin{array}{l}\text { Vorwurf an die Medien / Schimpf- } \\
\text { wort }\end{array}$ & 23 & 47 & 34 \\
$\begin{array}{l}\text { Synonym für die Medien } \\
\text { Unangebracht, weil Vokabular der }\end{array}$ & 36 & 10 & 24 \\
$\begin{array}{l}\text { Nationalsozialisten } \\
\text { Angebrachte Beschreibung der Me- }\end{array}$ & 10 & 5 & 8 \\
dien & 3 & 5 & 4 \\
$\begin{array}{l}\text { Sonstige Bewertung } \\
\text { Gesamt }\end{array}$ & 8 & 13 & 10 \\
\hline Annerkungen: $N$ (100 & 100 & 100 & 100 \\
\hline
\end{tabular}

Anmerkungen: $N=338 ; X^{2}=42.07, p<.001, \phi=.35$

Fazit

Der Begriff Lügenpresse ist zum Sinnbild für zwei aktuelle gesellschaftliche Phänomene geworden: Zum einen ein aggressiver werdender Kommunikationsstil überwiegend populistischer Akteurinnen, die damit den 
klassischen Massenmedien die Legitimität als Informationsquelle für die Bürgerinnen absprechen wollen. Zum anderen ist es ein Ausdruck eines Teils der Bevölkerung, der sich von den Massenmedien nicht (mehr) gut informiert und/oder schlecht repräsentiert fühlt. Auch wenn man, wie bereits diskutiert, nicht von einer Vertrauenskrise an sich sprechen kann, so lässt sich in den letzten Jahren dennoch eine zunehmende Polarisierung des Vertrauens in die klassischen Massenmedien beobachten. Auch wenn der Anteil derer, die den Massenmedien nicht oder nicht mehr vertrauen, die deutliche Minderheit darstellt, sollte man dieses Phänomen nicht trivialisieren. Man sollte stattdessen weiter untersuchen, welche Ursachen dahinterstecken.

Die Tatsache, dass sich die oft beklagte Vertrauenskrise in die Medien empirisch so gar nicht zeigen lässt, ist mitunter auch zweitrangig: Zahlreiche kommunikationswissenschaftliche Studien zu wahrgenommenen Medieneinflüssen konnten bereits zeigen, dass allein die Annahme, es könnte eine Vertrauenskrise in die Medien geben, handlungsrelevant sein könnte (siehe Forschung zu Third-Person-Effekt oder Influence of Presumed Media Influence).

Unser Interesse galt vor allem der Frage, inwieweit klassische Massenmedien, also genau die Ziele des Lügenpresse-Vorwurfs, eigentlich zur Verbreitung und eventuell auch Trivialisierung des Begriffs Lügenpresse beitragen. Stellvertretend haben wir uns dafür die Berichterstattung von fünf überregionalen Tageszeitungen von Oktober 2014 bis Oktober 2015 angesehen. Dabei sei gesagt, dass die hier präsentierten Befunde damit nicht das aktuellste Bild der Verwendung des Begriffs präsentieren, sondern ein Schlaglicht auf die Zeit werfen, in der der Ausdruck verstärkt wiederaufkam. Inwiefern sich der Umgang vier Jahre später davon unterscheidet, vermögen wir in diesem Beitrag nicht zu sagen.

Für die damalige Zeit lässt sich festhalten, dass der Begriff in den entsprechenden Tageszeitungen relativ unreflektiert - man möchte sagen unbedacht - verwendet wurde. In etwa der Hälfte der Verwendungsfälle wird der Begriff lediglich reproduziert, ohne dass irgendeine Form von Einordnung erfolgt - und sei es nur, dass es sich um unangemessene Pauschalkritik handelt. Eine stärkere Auseinandersetzung erfolgt vor allem da, wo einzelne Medienorganisationen oder -akteurinnen betroffen sind, gerade der pauschale Vorwurf an die Medien als Ganzes wird aber häufig einfach unkommentiert übernommen.

Dazukommt, dass es in der Mehrheit der Verwendungsfälle nicht so ist, dass der Begriff deshalb auftaucht, weil über einen Vorfall berichtet wird, bei dem der Begriff von jemanden verwendet wurde (zum Beispiel bei einer 
Kundgebung oder Demonstration), sondern die Autorin des Beitrags bringt das Wort Lügenpresse selbst ins Spiel (was wiederum nicht bedeutet, dass dies nicht ohne externen Kommunikationsanlass, wie etwa eine Demonstration geschieht). Zusammengenommen kann man also konstatieren, dass die Tageszeitungen zu einer Zeit, in der der Begriff gerade wieder in Mode kam, selbst dazu beigetragen haben, diesen größtenteils unreflektiert zu verbreiten.

Wie bereits bei Denner und Peter (2017) beschrieben, haben wir einige Vermutungen, warum Journalistinnen so unbedarft mit dem doch sehr harten Vorwurf umgehen. Ein Grund könnte sein, dass der Vorwurf eben als so ungerechtfertigt und übertrieben angesehen wird, dass eine Widerlegung oder gar Einordnung für das eigene Publikum gar nicht als nötig erachtet wird. Auch könnte eine geradezu ironische Verwendung als Synonym für die Medien bewusst verwendet werden, um den Begriff ins Lächerliche zu ziehen. Die Tatsache, dass der Ausdruck in genau dieser scherzhaften Weise (ähnlich wie der bekannte Ausspruch »Danke Merkel!«) auch des Öfteren von eher nicht medienkritischen Nutzerinnen in sozialen Netzwerken benutzt wird, erhärtet den Eindruck einer unreflektierten Übernahme des Begriffs durch das Publikum.

Eine häufige, unreflektierte Verwendung birgt aus unserer Sicht aber vor allem zwei Probleme: Zum einen steht zu befürchten, dass sich der Begriff dadurch trivialisiert und sich sprachlich als Ausdruck für die Massenmedien etabliert (beziehungsweise relativ schnell herangezogen wird, wenn zum Beispiel eine Zeitungsente produziert wird), was die Legitimation der Massenmedien beziehungsweise das Vertrauen in sie nicht zwangsläufig erhöhen dürfte. Zum anderen drückt gerade die ironische Verwendung durch die Medien selbst beziehungsweise ihnen nahestehende Akteurinnen und Bürgerinnen eine Missachtung derer aus, die durch diesen Begriff ihrem Misstrauen in die Medien Ausdruck verleihen wollen. Dies sollte man nicht abtun oder gar ins Lächerliche ziehen, sondern man sollte diese Bedenken ernst nehmen, ihre Ursachen erforschen und dem Vorwurf auf einer ernsten und sachlichen Ebene begegnen. 


\section{Literatur}

Allcott, Hunt \& Gentzkow, Matthew (2017). Social Media and Fake News in the 2016 Election. Journal of Economic Perspectives, 31, 211-236. DOI: 10.1257/ jep.31.2.211.

Beuthner, Michael \& Weichert, Stephan Alexander (2005). Zur Einführung. Internal Affairs - oder: die Kunst und die Fallen medialer Selbstbeobachtung. In: Beuthner, M. \& S. A. Weichert (Hrsg.), Die Selbstbeobachtungsfalle. Grenzen und Grenzgänge des Medienjournalismus (13-41). Wiesbaden: VS Verlag.

D'Angelo, Paul \& Esser, Frank (2014). Metacoverage and Mediatization in US Presidential Elections. A Theoretical Model and Qualitative Case Study. Journalism Practice, 8, 295-310. DOI: 10.1080/17512786.2014.889446.

Denner, Nora \& Peter, Christina (2017). Der Begriff Lügenpresse in deutschen Tageszeitungen. Eine Framing-Analyse. Publizistik, 62, 273-297. DOI: 10.1007/s11616017-0354-4.

Drentwett, Christine (2009). Vom Nachrichtenvermittler zum Nachrichtenthema. Metaberichterstattung bei Medienereignissen. Wiesbaden: VS Verlag.

Egelhofer, Jana Laura \& Lecheler, Sophie (2019). Fake News as a Two-Dimensional Phenomenon. A Framework and Research Agenda. Annals of the International Communication Association, 43, 97-116. DOI: 10.1080/23808985.2019.1602782.

Esser, Frank (2008). Metaberichterstattung. Medienselbstthematisierung und PublicityThematisierung in amerikanischen, britischen und deutschen Wahlkämpfen. In: Melischek, G.; J. Seethaler \& J. Wilke (Hrsg.), Medien \& Kommunikationsforschung im Vergleich - Grundlagen, Gegenstandsbereiche, Verfahrensweisen (121-156). Wiesbaden: VS Verlag.

Gniffke, Kai (2015). Wir bevormunden nicht. Wie inszeniert die »Tagesschau«? Die ARD antwortet. Frankfurter Allgemeine Sonntagszeitung, 41.

Hagen, Lutz (2015). Nachrichtenjournalismus in der Vertrauenskrise. »Lügenpresse« wissenschaftlich betrachtet. Journalismus zwischen Ressourcenkrise und entfesseltem Publikum. Communicatio Socialis, 48, 152-163. DOI: 10.5771/0010-3497-20152-152.

Heine, Matthias (2015). Man spricht rechts. Welt Online, 13. März 2015. Abgerufen von https://www.welt.de/print/die_welt/kultur/article138355134/Man-sprichtrechts.html.

Jackob, Nikolaus; Schultz, Tanjev; Jakobs, Ilka; Ziegele, Marc; Quiring, Oliver \& Schemer, Christian (2019). Medienvertrauen im Zeitalter der Polarisierung. Mainzer Langzeitstudie Medienvertrauen 2018. Media Perspektiven, 5, 210-220.

Jandura, Olaf \& Friedrich, Katja (2014). The quality of political media coverage. In: Reinemann, C. (Hrsg.), Political Communication (351-374). Berlin/Boston: De Gruyter Verlag.

Krüger, Udo Michael \& Müller-Sachse, Karl H. (1998). Medienjournalismus. Strukturen, Themen, Spannungsfelder. Opladen: Westdeutscher Verlag.

Malik, Maja (2004). Journalismusjournalismus. Funktion, Strukturen und Strategien der journalistischen Selbstthematisierung. Wiesbaden: VS Verlag. 
Müller, Philipp \& Denner, Nora (2019). Was tun gegen »Fake News«? Eine Analyse anhand der Entstehungsbedingungen und Wirkweisen gezielter Falschmeldungen im Internet. Gutachten im Auftrag der Friedrich-Naumann-Stiftung für die Freiheit.

Nelson, Jacob L. \& Taneja, Harsh (2018). The Small, Disloyal Fake News Audience. The Role of Audience Availability in Fake News Consumption. New Media \& Society, 20(10), 3720-3737. DOI: 10.1177/1461444818758715.

Neverla, Irene (2017). »Lügenpresse« - Begriff ohne jede Vernunft? Eine alte Kampfvokabel in der digitalen Mediengesellschaft. In: Lilienthal, V. \& I. Neverla (Hrsg.), Lügenpresse. Anatomie eines politischen Kampfbegriffs (18-44). Köln: Kiepenheuer \& Witsch.

Reinemann, Carsten \& Fawzi, Nayla (2016). Eine vergebliche Suche nach der Lügenpresse. Der Tagesspiegel Online, 24. Januar 2016. Abgerufen von https://www. tagesspiegel.de/politik/analyse-von-langzeitdaten-eine-vergebliche-suche-nach-derluegenpresse/12870672.html.

Reinemann, Carsten \& Huismann, Jana (2007). Beziehen sich Medien immer mehr auf Medien? Dimensionen, Belege, Erklärungen. Publizistik, 52, 465-484. DOI: 10.1007/ s11616-007-0243-3.

Reinemann, Carsten; Fawzi, Nayla \& Obermaier, Magdalena (2017). Die »Vertrauenskrise« der Medien - Fakt oder Fiktion? In: Lilienthal, V. \& I. Neverla (Hrsg.), Lügenpresse. Anatomie eines politischen Kampfbegriffs (77-94). Köln: Kiepenheuer $\&$ Witsch.

Ronneberger, Franz (1974). Die politischen Funktionen der Massenkommunikation. In: Langenbucher, W. (Hrsg.), Zur Theorie der politischen Kommunikation (193-206). München: Piper.

Tandoc, Edson C.; Lim, Zheng Wei \& Ling, Richard (2018). Defining »fake news«. A typology of scholarly definitions. Digital Journalism, 6, 137-153. DOI: 10.1080/21670811.2017.1360143.

Torres, Russell; Gerhart, Natalie \& Negahban, Arash (2018). Combating fake news. An investigation of information verification behaviors on social networking sites. Proceedings of the 51st Hawaii International Conference on System Sciences. DOI: 10.24251/HICSS.2018.499.

Ziegele, Marc; Schultz, Tanjev; Jackob, Nikolaus; Granow, Viola; Quiring, Oliver \& Schemer, Christian (2018). Lügenpresse-Hysterie ebbt ab. Mainzer Langzeitstudie »Medienvertrauen«. Media Perspektiven, 4, 150-162.

Zimmermann, Fabian \& Kohring, Matthias (2018). »Fake News« als aktuelle Desinformation. Systematische Bestimmung eines heterogenen Begriffs. Medien \& Kommunikationswissenschaft, 66, 526-541. DOI: 10.5771/1615-634X-2018-4-526 